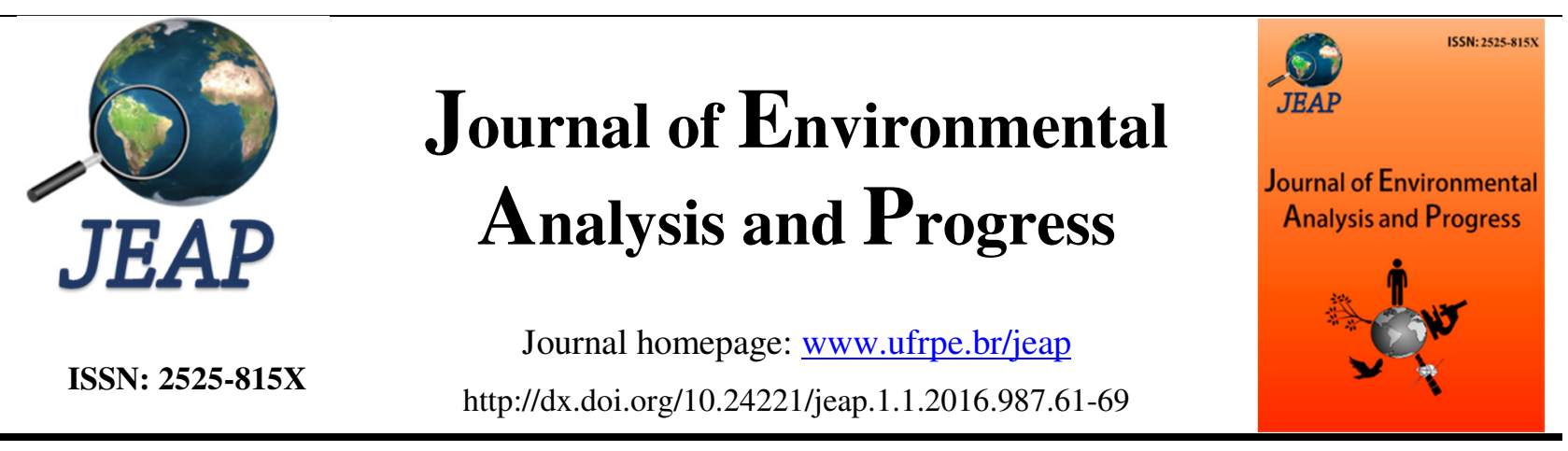

\title{
Banco de sementes em áreas de caatinga com diferentes graus de antropização
}

\section{Seed bank in areas of savanna with different degrees of human disturbance}

\author{
Gyslainne Vanderley da Paz ${ }^{\mathrm{a}}$, Kleber Andrade da Silva ${ }^{\mathrm{b}}$, Jarcilene Silva Almeida-Cortez ${ }^{\mathrm{c}}$ \\ a Universidade Federal de Pernambuco-UFPE, Departamento de Botânica-DB, Centro de Biociências-CB, Av. Prof. \\ Moraes Rego, 1235. Cidade Universitária, Recife, Pernambuco, Brasil. CEP: 50670-901

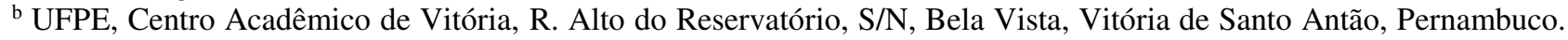 \\ CEP: 55608-680, Brasil. \\ ${ }^{\text {c } U F P E-D B-C B . ~ E-m a i l: ~ j a c o r t e z @ u f p e . b r . ~}$
}

\section{A R T I CLE IN F O}

Recebido 29 Sep 2016

Aceito 11 Oct 2016

Publicado 13 Oct 2016

\begin{abstract}
A B S T R A C T
Arid and semi-arid area characterizes the Caatinga due to its irregularity of precipitation and characterized by long periods of drought. The investigation of the seed bank in these regions allows predicting a future scenario of plant communities on anthropogenic pressures, in addition to the information about the current flora. This study aims at comparing the density and floristic composition of the seed bank of the seeds accumulated during the dry season from different areas of Caatinga. Fourteen areas of Caatinga were selected according to the degree of human disturbance, water gradient and different intensities of herbivory at Floresta and Itacuruba, both at Pernambuco. We collected five samples of litter in 14 plots. The material was taken to the greenhouse of the UFPE, arranged directly in trays and watered daily and observed up three months. The peak of germination was in the first four weeks of the material from all plots. We identified forty-four seedlings morphospecies of grass, herbaceous and tree. The highest richness and most abundant species germinated was the herbs species. There was no significant difference between species richness and herbivory pressure according to GLM analysis, but there was significant difference between density and herbivory pressure at all distances from watercourses and NMDL analysis it was observed that there was only difference significant between areas with permanent watercourse and areas without a nearby watercourse (Rglobal $=0.155 ; \mathrm{p}=0.014)$.
\end{abstract}

Keywords: Plant community, land use history, Tropical Dry Forest.

\begin{abstract}
R E S U M O
A caatinga é caracterizada por regiões áridas e semi-áridas devido a sua irregularidade de precipitação e caracterizada por longos períodos de estiagem. A investigação do banco de semente presente nessas regiões, além de nos dar informações sobre a flora atual, possibilita predizer um cenário futuro das comunidades vegetais diante de pressões antrópicas. Este trabalho tem por objetivo analisar comparativamente a densidade e a composição florística do banco de sementes acumuladas da estação seca provenientes de diferentes áreas de caatinga. Foram selecionadas 14 áreas de caatinga de acordo com grau de antropização, gradiente hídrico e diferentes intensidades de herbivoria nos municípios de Floresta e Itacuruba, ambos em Pernambuco. Foram coletadas cinco amostras de serrapilheira, em 14 parcelas. O material foi levado para casa de vegetação na UFPE, dispostos diretamente em bandejas de isopor, com regas diárias e observado por três meses. Foi observado um pico de germinação nas primeiras quatro semanas para o material oriundo de todas as parcelas. Foram identificadas 44 morfoespécies de
\end{abstract}


plântulas de gramíneas, herbáceas e arbóreas. A maior riqueza de espécies germinadas foi de herbáceas, bem como as mais abundantes. Não houve diferença significativa entre riqueza de espécies e pressão de herbivoria segundo a análise de GLM, mas houve diferença significativa entre a densidade e pressão de herbivoria para todas as distâncias de cursos de água e a partir da análise de NMDL foi observado que só houve diferença significativa entre áreas com curso permanente de água e áreas sem um curso de água próximo $\left(\mathrm{R}^{\text {global }}=0.155 ; \mathrm{p}=0,014\right)$.

Palavras-Chave: Comunidade vegetal, histórico de uso da terra, Floresta Tropical Seca.

\section{Introdução}

As florestas tropicais secas representam a terceira maior formação vegetal dos trópicos, em área segundo Sabogal (1992). Quando comparadas a outras formações vegetais, as florestas tropicais secas são as que mais apresentam perda de sua vegetação devido às atividades humanas. De acordo com Powers et al. (2011), foi observado que metade da vegetação natural, de quase $80 \%$ das florestas tropicais secas, foi perdida por causas antrópicas. Aproximadamente $42 \%$ das florestas tropicais de todo mundo são de florestas tropicais secas, onde mais da metade ocorrem nas Américas (Murphy \& Lugo, 1986; Portillo-Quintero \& Sánchez-Azofeifa, 2010); no Brasil, está representada pela caatinga.

A província das Caatingas no nordeste do Brasil estende-se de $2^{\circ} 54^{\prime}$ a $17^{\circ} 21^{\prime}$ 'S (estimada em cerca de $800.000 \mathrm{~km}^{2}$ pelo IBGE, em 1985) e inclui os estados do Ceará, Rio Grande do Norte, a maior parte da Paraíba e Pernambuco, sudeste do Piauí, oeste de Alagoas e Sergipe, região norte e central da Bahia e uma faixa estendendo-se ao norte de Minas Gerais seguindo o rio São Francisco, juntamente com um enclave no vale seco da região média do rio Jequitinhonha (Andrade-Lima, 1981). As áreas de vegetação de caatinga, em geral, vêm sofrendo muita perturbação antrópica, sendo frequentemente utilizadas para o estabelecimento de atividades de agricultura ou utilizadas como áreas de pastagens nativas (Araújo et al., 2007). Apesar da importância da existência de um banco de sementes para a regeneração de áreas antropizadas (Almeida-Cortez, 2004), até o momento, o banco de sementes de áreas de caatinga só foi estudado apenas em quatro áreas consideradas preservadas pelos pesquisadores (Costa \& Araújo, 2003; Pessoa, 2007; Mamede \& Araújo, 2008; Lobo, 2008).

O banco de sementes pode ser definido como sendo o estoque de sementes viáveis existentes no solo desde a superfície até as camadas mais profundas, em uma dada área e em um dado momento. $\mathrm{O}$ acúmulo de sementes no banco varia de acordo com a entrada (dispersão) e saída (germinação, morte) de sementes (Baker, 1989; Roberts \& Simpson, 1989; Almeida-Cortez, 2004). O banco pode ser formado por sementes alóctomas (originais de outros locais) e/ou autóctomas (sementes das espécies locais) (Almeida-Cortez,
2004). O predomínio de sementes de espécies herbáceo-arbustivo presente no solo das florestas tropicais pode ser uma importante fonte de recrutamento de plântulas após alguma perturbação e auxiliar na regeneração das florestas (AlmeidaCortez, 2004).

A composição do banco de sementes varia o longo das estações do ano. Além disso, em função da longevidade dos diásporos, os bancos de sementes podem ser caracterizados como transitórios, ou seja, formados por sementes de curta viabilidade, e persistentes, compostos por sementes de maior longevidade sob condições naturais (AlmeidaCortez, 2004).

O banco de sementes do solo de uma floresta representa o estoque de sementes não germinadas que, quando as condições favoráveis se apresentam, são capazes de substituir as plantas que eventualmente tenham desaparecido do ecossistema (Souza et al., 2006). As sementes são adicionadas ao banco de sementes do solo continuamente, através de dispersão e chuva de sementes. Essas sementes podem germinar logo após a sua dispersão ou entrarem em dormência e se incorporarem ao banco (Santos, 2000).

Em geral, as espécies que formam banco de sementes no solo, como estratégia de manter-se na área, apresentam mecanismos eficientes de dispersão de sementes, produção abundante, bem como estas apresentam dormência e longevidade elevada. Estas características são comumente observadas nas espécies pioneiras, enquanto as espécies secundárias tardias e clímax tendem a formar bancos de plântulas, uma vez que suas sementes apresentam baixa viabilidade e acentuada predação. (PiñaRodrigues, Costa \& Reis, 1992; Costalonga, 2006).

Este estudo objetivou analisar, comparativamente, a densidade e a composição florística do banco de sementes acumuladas da estação seca e germinadas em casa de vegetação oriundas de diferentes áreas de caatinga, a partir de um gradiente hídrico e diferentes intensidades de herbivoria.

\section{Material e Métodos}

Área de estudo

As áreas de coleta se localizam nos municípios de Itacuruba (843'39.4’S, 3841'05.2”W) e Floresta 
(8³6'08.9'S, 38³4'15.4'W), localizados na mesorregião de Itaparica, Pernambuco (Figura 1).

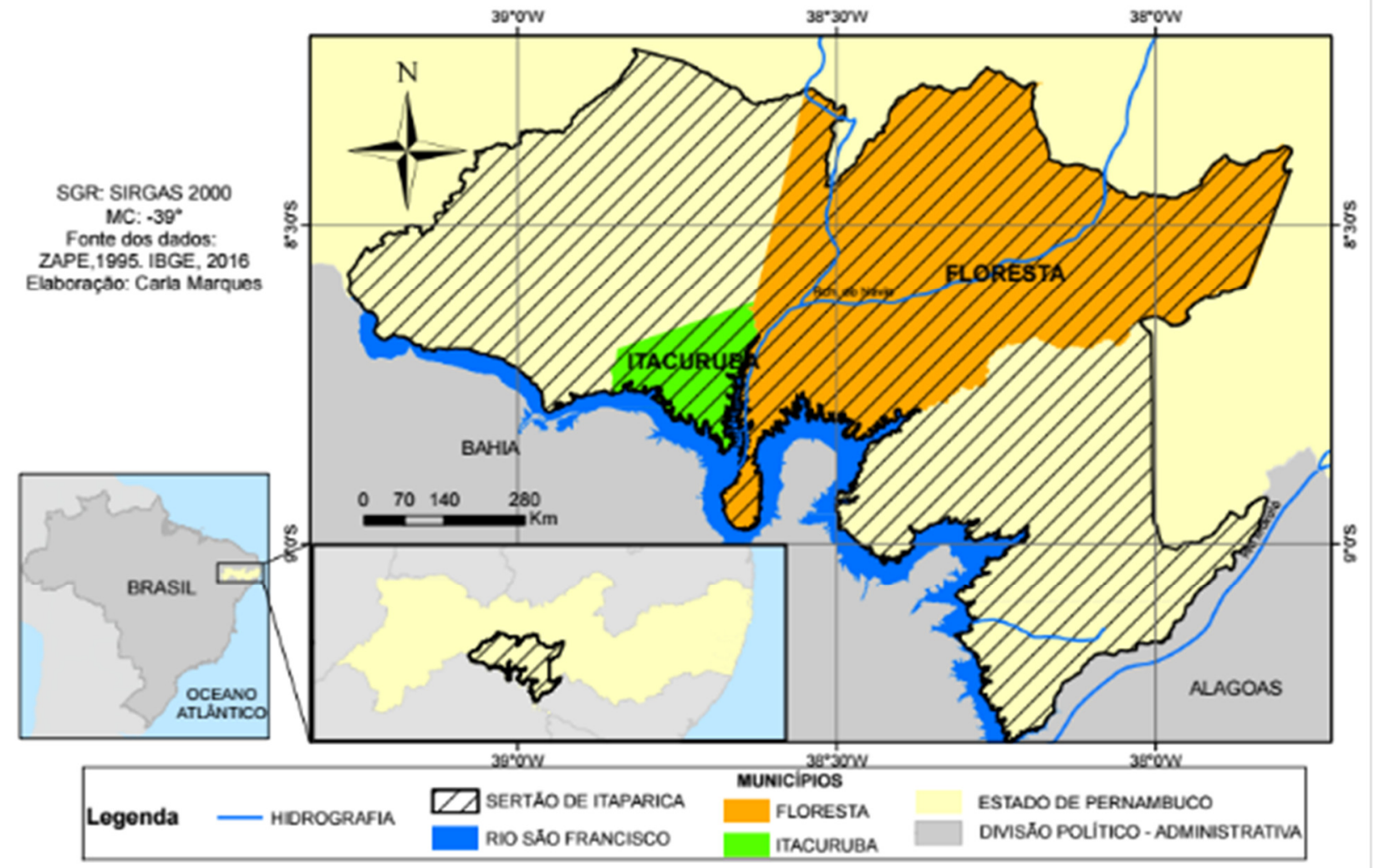

Figura 1. Mapa da localização dos municípios de Floresta e Itacuruba, Pernambuco, Brasil.

Em 2015, ano da coleta de dados para a realização do estudo, os índices pluviométricos acumulados foram de $96,5 \mathrm{~mm}$ e $223,5 \mathrm{~mm}$ para
Itacuruba e Floresta, Pernambuco, respectivamente (Figura 2).

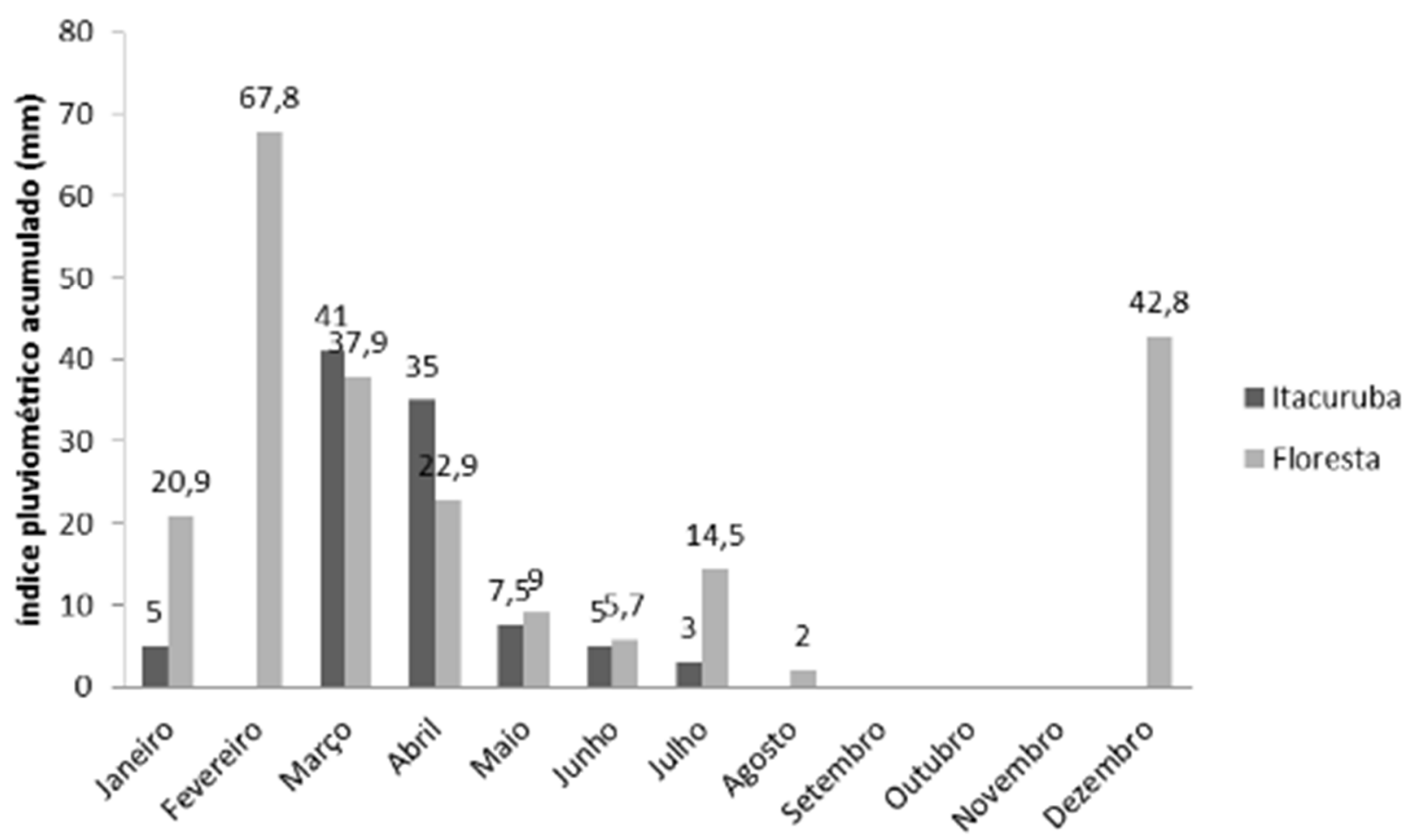

Figura 2. Índice pluviométrico mensal, em milímetros, dos municípios de Itacuruba e Floresta, Pernambuco do ano de 2015. Fonte: Instituto Agronômico de Pernambuco. 


\section{Desenho experimental}

Foram utilizados dois critérios de estratificação para cobrir grandes gradientes de intensidade de pastejo e disponibilidade de água. As parcelas foram estratificadas em três diferentes classes de intensidade de pastejo com base em avaliação visual: pastagem com alta pressão (parcelas localizadas em pastagens), pressão de pastejo intermediária (parcelas localizadas na floresta caatinga não utilizada) e caatinga preservada (áreas sem sinais claros de pastagem).

A área de estudo foi ainda estratificada em três diferentes classes de disponibilidade de água (Parcelas localizadas na margem do lago dentro de 5 $\mathrm{m}$ do nível de água elevado, parcelas ao longo de cursos de água temporários e parcelas sem qualquer sinal de água de superfície nas imediações) (Schulz et al., 2016). A intensidade de pastejo foi avaliada segundo a metodologia de Schulz et al. (2016).

A coleta foi realizada em janeiro de 2015, no final do período seco; nesse período não houve precipitação nos municípios de Floresta e Itacuruba, de acordo com os dados pluviométricos coletados pelo Instituto Agronômico de Pernambuco (IPA).
Foram coletadas cinco amostras de serapilheira em cada um dos 14 pontos de amostragem (parcelas) $50 \times 50 \mathrm{~m}$ dispostas na forma de "L", totalizando em 70 amostras (Figura 3).

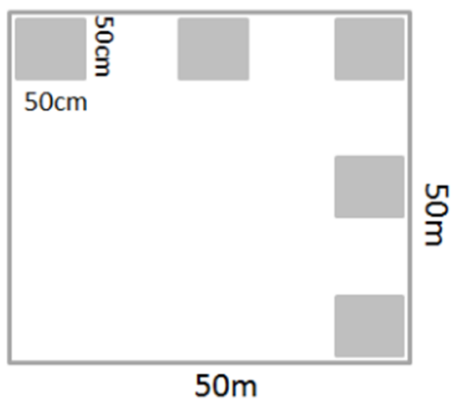

Figura 3. Esquema representativo de uma parcela e seus 5 pontos de coleta em forma de " $\mathrm{L}$ " $\operatorname{com} 50 \mathrm{~cm}^{2}$.

As regiões de coleta foram classificadas de acordo com a disponibilidade hídrica, como regiões com escassez de água, regiões com curso de água temporário e regiões com água permanente, sob alta ou baixa pressão de herbivoria (Figura $4 \mathrm{ABC}$ ).

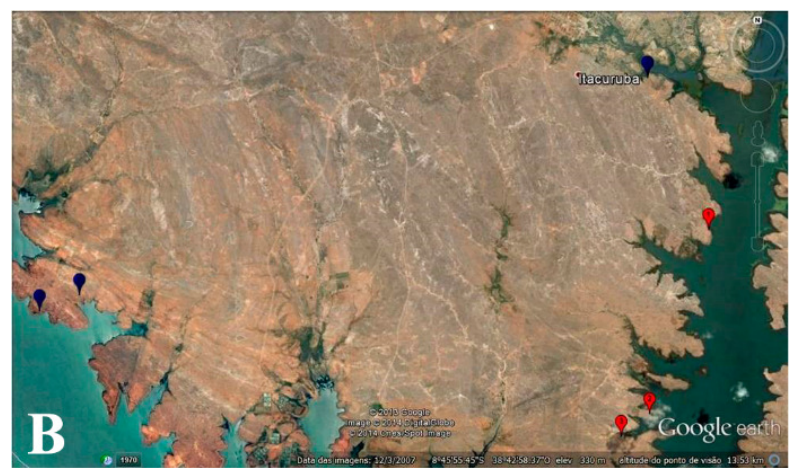

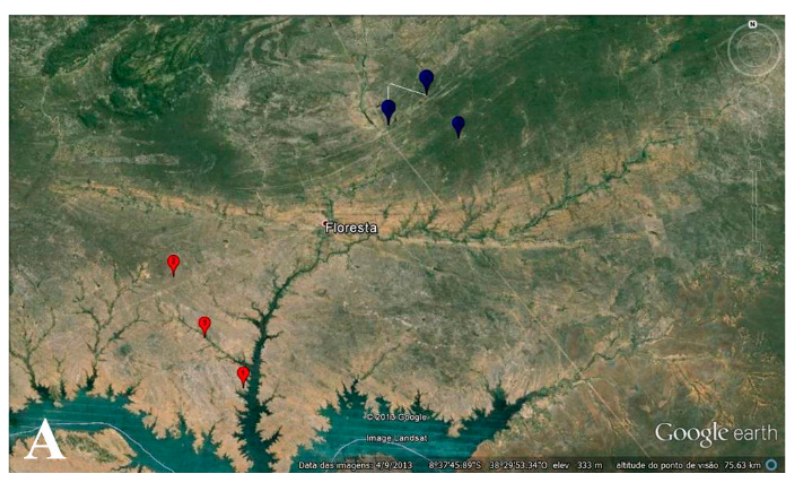

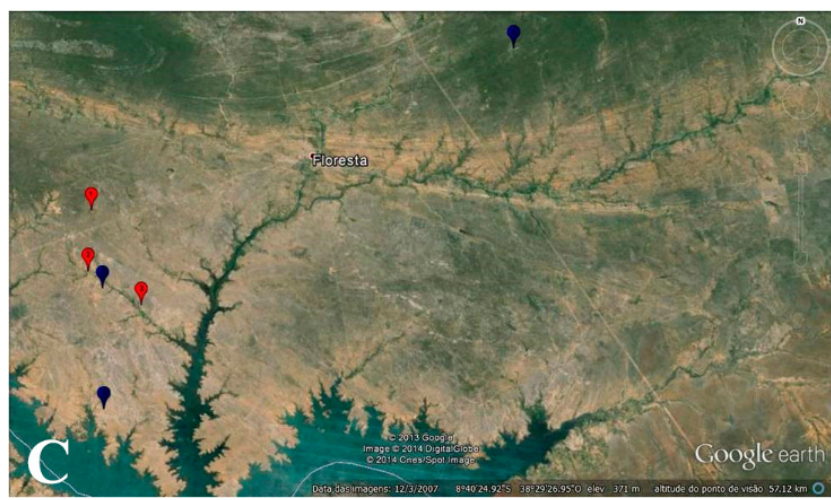

Figura 4. Região sem a presença de um curso de água próximo (A), região com curso de água permanente (B) e região com curso de água temporária $(\mathrm{C})$, em vermelho, áreas que sofrem alta pressão de herbivoria, em azul, áreas que sofrem baixa pressão de herbivoria.

A coleta das amostras de serapilheira foi realizada com o auxílio de um quadrado de PVC $(50 \mathrm{~cm} \times 50 \mathrm{~cm})$ para demarcar a área de coleta e com o auxílio de uma pá de jardinagem, o material foi acondicionado em sacos plásticos e levados para a casa de vegetação do Departamento de Botânica, no Centro de Biociências (CB) da Universidade Federal de Pernambuco (UFPE). As amostras foram triadas a fim de retirar pedregulhos e dispostos em bandejas 
de isopor $(26,5 \mathrm{~cm} \times 26,5 \mathrm{~cm} \times 0,5 \mathrm{~cm})$, devidamente identificadas.

Entre as bandejas foram distribuídas cinco bandejas controle, contendo areia lavada, a fim de monitorar possível contaminação das amostras (Figura 5). A rega foi diária e foram realizadas observações por um período de três meses.
As plântulas foram marcadas e transferidas para acompanhar o desenvolvimento até ser possível a identificação taxonômica das espécies. No caso das herbáceas, a rega estendeu-se até a fase reprodutiva. $\mathrm{O}$ material foi revolvido sempre que passavam alguns dias sem a detecção de novas sementes germinadas.

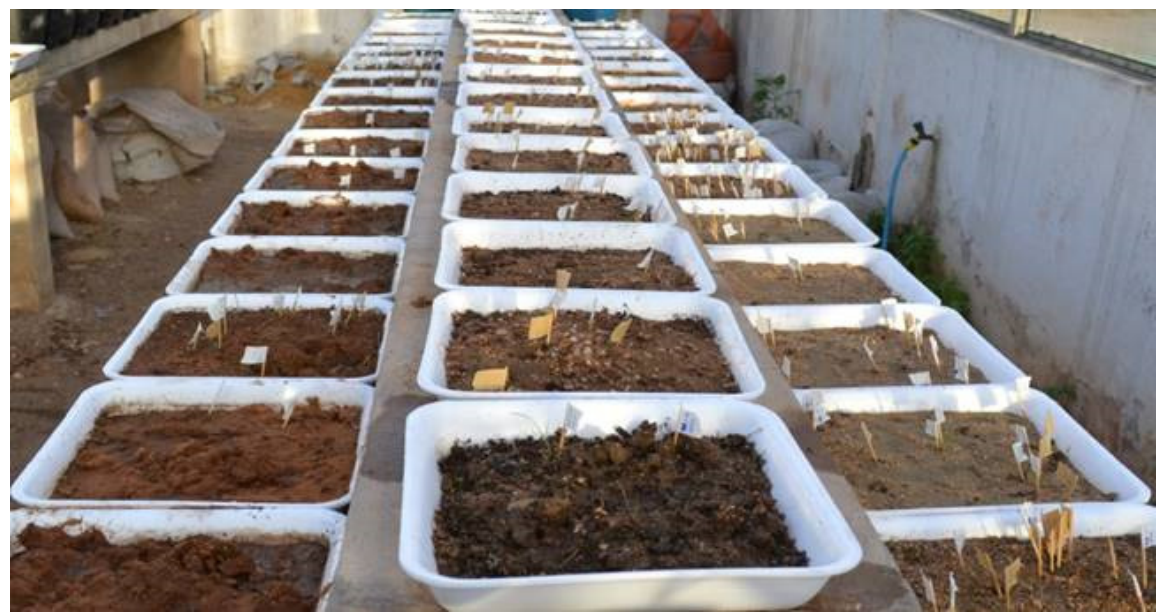

Figura 5. Disposição das amostras de serrapilheira em bandejas de isopor em casa de vegetação da Universidade Federal de Pernambuco (UFPE).

\section{Análise estatística}

O poder explicativo da distância de um curso de água e o grau de herbivoria e suas interações sobre a riqueza de espécies e densidade na estação seca foi avaliada utilizando o modelo linear geral (GLM).

As diferenças na riqueza de espécies e densidade entre as áreas com curso de água permanente, rio temporário e sem presença de um curso de água próximo em alto ou baixo grau de herbivoria foram verificadas pela Tukey um teste posteriori. Todas estas análises foram realizadas utilizando o software estatístico 7,0 (StatSoft Inc, 2003).

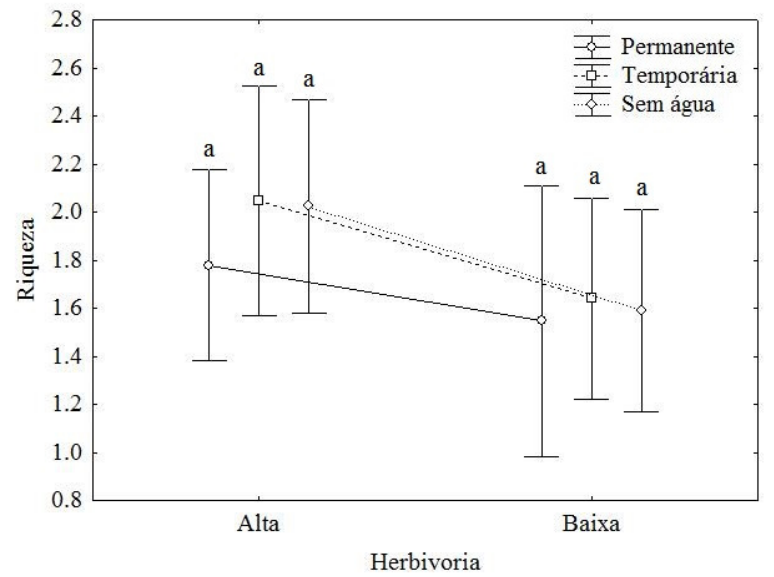

\section{Resultados}

Foram identificadas 4.053 sementes germinadas no banco de sementes para as três áreas de coleta, $35,23 \%$ do total proveniente do banco de sementes das áreas com curso de água permanente, $28,27 \%$ das áreas com curso de água temporária, e $36,49 \%$ das áreas sem a presença de curso de água.

Houve um pico de germinação nas 4 primeiras semanas de observação.

Não houve diferença significativa na análise entre riqueza de espécies e pressão de herbivoria (Figura 6A). Houve diferença significativa na análise entre a densidade e pressão de herbivoria para todas as distâncias de curso de água (Figura 6B).

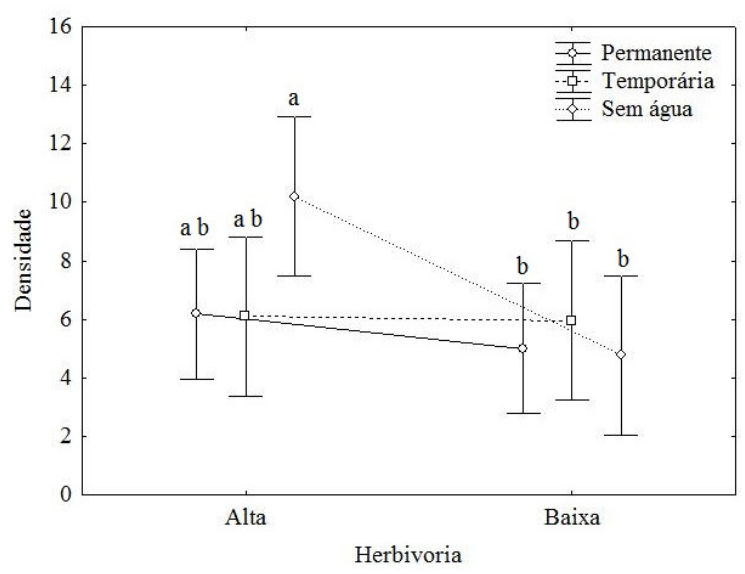

Figura 6. Variação de riqueza média (número de espécies por parcela) em "A" e média da densidade de sementes (sementes por parcela) em "B", em áreas com curso de água permanente, rio temporário e sem a presença de um curso de água próximo em relação a herbivoria. As barras verticais indicam o intervalo de confiança de 0,95 . 
Foram germinadas sementes de 44 morfoespécies, destas, 31 foram identificadas distribuídas em 17 famílias (Tabela 1). As espécies Portulaca elatior Mart e $P$ oleracea L. Apresentaram maior densidade de indivíduos germinados, ambas consideradas espécies nativas de acordo com o herbário virtual - REFLORA.

Quanto ao hábito das espécies identificadas, as herbáceas são o grupo mais representativo, com $80,65 \%$ do total, englobando 25 espécies. Os subarbustos e árvores representam respectivamente $16,13 \%$ e $3,23 \%$ do total.

As composições florísticas de ambas as áreas, com curso de água permanente, rio temporário e sem a presença de um curso de água próximo, comparados por uma análise de escalonamento multidimensional não-métrico (NMDS) usando a matriz de dissimilaridade Brayn e Curtis, com base na densidade relativa das espécies nas 70 parcelas. A análise de semelhanças (ANOSIM) foi usada para testar o significado do grupo formado na NMDS. Todas estas análises foram realizadas usando a versão do software Primer 6.1.6 (Clarke \& Gorley, 2006).

A partir da análise de NMDS foi verificado que no geral não houve diferença significativa entre as áreas $\left(\mathrm{R}^{\text {global }}=0.063 ; \mathrm{p}=0,058\right.$ entre as áreas (Figura 7). Comparando as áreas par a par não houve diferença significativa entre as áreas com curso de água permanente e com curso de água temporária $\left(R^{\text {global }}=0.002 ; p=0,38\right)$ nem entre as áreas sem curso de água próximo e com curso de água temporária $\left(\mathrm{R}^{\text {global }}=0.029 ; \mathrm{p}=0,21\right)$, houve diferença apenas nas áreas com curso de água permanente e sem curso de água próximo $\left(\mathrm{R}^{\text {global }}=0.155 ; \mathrm{p}=0,014\right)$. A diferença entre essas duas áreas explicou $15,5 \%$ da diferença da composição florística, é diferente, mas esse percentual ainda é considerado baixo.

Tabela 1. Composição florística do banco de sementes do período seco em uma região da caatinga dos municípios de Floresta e Itacuruba, Pernambuco, com seus respectivos hábitos, síndromes de dispersão e origem.

\begin{tabular}{|c|c|c|c|c|}
\hline Família & Espécie & Hábito & $\begin{array}{l}\text { Síndrome de } \\
\text { dispersão* }\end{array}$ & Origem \\
\hline Amaranthaceae & Alternanthera tenella Colla & Subarbusto & Anemocórica & Nativa \\
\hline \multirow[t]{2}{*}{ Boraginaceae } & Euploca procumbens (Mill.) Diane \& Hilger & Subarbusto & Zoocórica & Nativa \\
\hline & Heliotropium indicum $\mathrm{L}$ & Herbácea & Zoocórica & Nativa \\
\hline Cactaceae & Cactaceae sp. & - & - & - \\
\hline Cleomaceae & Cleome guianensis Aubl. & Herbácea & - & Nativa \\
\hline \multirow[t]{5}{*}{ Cyperaceae } & Cyperus odoratus L. & Herbácea & - & Nativa \\
\hline & Cyperus pelophilus Ridl. & Herbácea & - & Nativa \\
\hline & Eleocharis geniculata (L.) Roem. \& Schult. & Herbácea & - & Nativa \\
\hline & Fimbristylis sp. & Herbácea & - & - \\
\hline & Lipocarpha micrantha (Vahl) G.C.Tucker & Herbácea & - & Nativa \\
\hline \multirow[t]{2}{*}{ Euphorbiaceae } & Chamaesyce thymifolia (L.) Millsp. & Herbácea & - & Nativa \\
\hline & Chamaesyce hyssopifolia (L.) Small & Herbácea & - & Nativa \\
\hline Fabaceae & Prosopis juliflora (Sw.) DC. & Arbórea & Zoocórica & Exótica \\
\hline \multirow[t]{2}{*}{ Lamiaceae } & Hyptis suaveolens (L.) Poit & Herbácea & Anemocórica & Nativa \\
\hline & Marsypianthes chamaedrys (Vahl) Kuntze & Subarbusto & Autocórica & Nativa \\
\hline \multirow[t]{2}{*}{ Malvaceae } & Waltheria bracteosa A.St.-Hil. \& Naudin & Subarbusto & - & Nativa \\
\hline & Waltheria rotundifolia Schrank & Subarbusto & - & Nativa \\
\hline Molluginaceae & Mollugo verticillata $\mathrm{L}$. & Herbácea & - & Nativa \\
\hline Nyctaginaceae & Boerhavia diffusa L. & Herbácea & Zoocórica & Naturalizada \\
\hline Onagraceae & Ludwigia octovalvis (Jacq.) P. H. Raven & Herbácea & Autocórica & Nativa \\
\hline \multirow[t]{2}{*}{ Phyllanthaceae } & Phyllanthus amarus Schumach. & Herbácea & Autocórica & Nativa \\
\hline & Phyllanthus heteradenius Müll. Arg. & Herbácea & Autocórica & Nativa \\
\hline Phytolaccaceae & Microtea paniculata Moq. & Herbácea & - & Nativa \\
\hline \multirow[t]{4}{*}{ Poaceae } & Dactyloctenium aegyptium (L.) Willd. & Herbácea & Anemocórica & Naturalizada \\
\hline & Eragrostis pilosa (L.) P.Beauv. & Herbácea & Anemocórica & Naturalizada \\
\hline & Enteropogon mollis (Nees) Clayton & Herbácea & Anemocórica & Nativa \\
\hline & Melinis repens (Willd.) Zizka & Herbácea & Anemocórica & Naturalizada \\
\hline
\end{tabular}


Journal of Environmental Analysis and Progress V. 01 N. 01 (2016) 61-69

\begin{tabular}{lclcc} 
& Tragus berteronianus Schult. & Herbácea & Anemocórica & Nativa \\
Portulacaceae & Portulaca elatior Mart. & Herbácea & Anemocórica & Nativa \\
Rubiaceae & Portulaca oleracea L. & Herbácea & Anemocórica & Nativa \\
& Diodella teres (Walter) Small & Herbácea & - & Nativa \\
& Mitracarpus longicalyx E.B.Souza \& & Herbácea & - & Nativa \\
\hline
\end{tabular}

*Informação obtida em Lima \& Melo (2015).

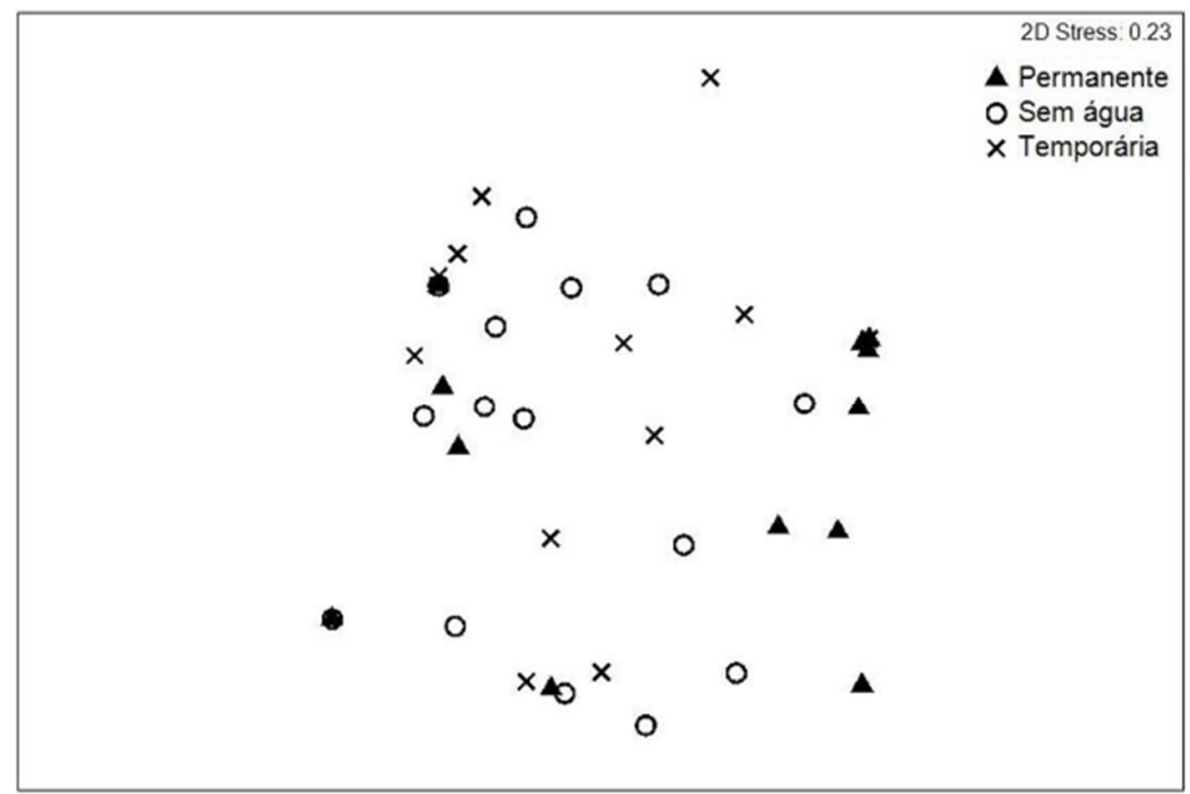

Figura 7. Ordenação formada após escalonamento multidimensional não-métrico análise (NMDS) com base em Bray-Curtis coeficientes de similaridade de espécies. Este gráfico foi produzido com base na riqueza de espécies por área. Os símbolos no gráfico representam as parcelas e suas respectivas espécies de abundância.

\section{Discussão}

O pico de germinação ocorrido nas quatro primeiras semanas pode indicar que as sementes presentes no banco apresentam uma rápida resposta ao início da rega, simulando as chuvas, dada à sazonalidade e a irregularidade do regime pluviométrico, provavelmente o banco de sementes da caatinga apresenta características similares às observadas em regiões desérticas e semi-desérticas, ou seja, sementes com alta germinabilidade no início da estação chuvosa. As espécies identificadas neste estudo são apontadas pela literatura como presentes em áreas de caatinga (veja Lima \& Melo, 2015).

Quanto ao hábito das espécies presentes no banco predominância de indivíduos pertencentes ao extrato herbáceo explica esse tipo de resposta, pois por apresentarem um ciclo de vida mais curto, precisam germinar logo ao início das chuvas, para assegurar uma nova geração de sementes e manter populações viáveis (Costa \& Araújo, 2003). Assim, estes indivíduos ganham vantagem em ambientes como o da Caatinga, onde a precipitação anual é baixa e bastante irregular (Mamede \& Araújo, 2008; Santos et al., 2011). Este padrão de germinação foi visto por Costa \& Araújo (2003) e Mamede e Araújo (2008), ambos trabalhando com banco de sementes em área de caatinga e também por Araújo et al. (2004) em uma área de Floresta estacional decidual ripária com influência sazonal, no Rio Grande do Sul.

\section{Conclusão}

O impacto da herbivoria nestas áreas de caatinga não interfere na riqueza de espécies, mas interfere na densidade de sementes disponíveis no solo, o que pode ser explicado pela compactação do solo provocada pelo pisoteio animal.

\section{Agradecimentos}

À Fundação de Amparo a Ciência e Tecnologia de Pernambuco-FACEPE pelo auxílio financeiro (APQ-0842-2.05/12) e concessão de bolsa de Iniciação Científica à primeira autora (BIC-17462.05/14). Este projeto faz parte projeto INNOVATE, financiado pela MCTI (Proc. N. 490003/2012-5). Ao Conselho Nacional de Desenvolvimento Científico e Tecnológico-CNPq pela concessão da Bolsa Produtividade (PQ-307422/2012-7) à AlmeidaCortez. Agradeço à Ligia Gomes, Fernanda Tavares, João Gomes, Bruno Lustosa, Silvia Santos, Fernando Sena pelo apoio logístico nas atividades de campo. 


\section{Referências}

ALMEIDA-CORTEZ, J. S. 2004. Dispersão e banco de sementes. In: FERREIRA, A. G.; BORGHETTI, F. (Orgs.). Germinação: do básico ao aplicado, Recife. pp.225-235.

ANDRADE-LIMA， D. 1981. The Caatingas Dominium. Revista Brasileira de Botânica, v.4, p.149-153.

ARAÚJO, E. E.; CASTRO, C. C; ALBUQUERQUE, U. P. 2007. Dynamics of Brazilian caatinga - A review concerning the plants, environment and people. Functional Ecosystems and Communities, v.1, p.15-28.

ARAÚJO, M. M.; LONGHI, S. J.; BARROS, P. L. C.; BRENA, D. A. 2004. Caracterização da chuva de sementes, banco de sementes do solo e banco de plântulas em Floresta Estacional Decidual ripária Cachoeira do Sul, RS, Brasil. Scientia Forestalis, v.66, p.128-141.

BAKER, H. G. 1989. Some aspects of the natural history of seed banks. pp.9-21. In: LECK, M. A.; PARKER, T. V.; SIMPSON, R. L. A. F. (Eds.). Ecology of soil seed banks, New York.

CLARKE, K. R.; GORLEY, R. N. 2006. Primer v6: User Manual/tutorial. Plymouth Marine Laboratory, Plymouth.

COSTA, R. C. D.; ARAÚJO, F. S. D. 2003. Densidade, germinação e flora do banco de sementes no solo, no final da estação seca, em uma área de caatinga, Quixadá, CE. Acta Botanica Brasilica, v.17, p.259-264.

COSTALONGA, S. R. 2006. Banco de sementes em áreas contíguas de pastagem degradada, plantio de eucalipto e floresta natural, em Paula Cândido - MG. 126f. Tese (Doutorado em Ciência Florestal) Universidade Federal de Viçosa.

IBGE. 1985. Atlas Nacional do Brasil. Região Nordeste. Fundação Instituto Brasileiro de Geografia e Estatística, Rio de Janeiro.

LIMA, E. A.; MELO, J. I. M. 2015. Biological spectrum and dispersal syndromes in an area of the semi-arid region of north-eastern Brazil. Acta Scientiarum, v. 37, n. 1, p. 91-100.

LOBO, P.C.A 2008 Análise do banco de sementes de uma área de caatinga-PE após simulação de seca. Monografia (Bacharelado em Ciências Biológicas) Universidade de Pernambuco, Recife. 34 p.
MURPHY, P. G.; LUGO, A. E. 1986. Ecology of Tropical Dry Forest. Annual Review of Ecology and Systematics, v.17, p.67-88.

MAMEDE, M. A.; ARAÚJO F. S. 2008. Effects of slash and burn practices on a soil seed bank of caatinga vegetation in Northeastern Brazil. Journal of Arid Environments, v.72, p.458-470.

PESSOA, L. M. 2007. Variação espacial e sazonal do banco de sementes do solo em uma área de caatinga, Serra Talhada, PE. Dissertação (Mestrado em Botânica), Universidade Federal Rural de Pernambuco, Recife. 45 p.

PIÑA-RODRIGUES, F. C. M.; COSTA, L. G. S.; REIS, A. 1992. Estratégias de estabelecimento de espécies arbóreas e o manejo de florestas tropicais. In: Congresso Florestal Brasileiro, 6, Campos do Jordão. Anais... São Paulo, 1992. p.676-684.

PORTILLO-QUINTERO, C. A.; SÁNCHEZAZOFEIFA, G. A. 2010. Extent and conservation of tropical dry forests in the Americas. Biological Conservation, v.143, p.144-155.

POWERS, J. S., CORRE, M. D., TWINE, T. E., VELDKAMP, E. 2011. Geographic bias of field observations of soil carbon stocks with tropical landuse changes precludes spatial extrapolation. Proceedings of the National Academy of Science of the United States of America, v.108, p.6318-6322.

HERBÀRIO VIRTUAL - REFLORA. Disponível em: http://reflora.jbrj.gov.br/reflora/herbarioVirtual I. Acesso em: 20/9/2016.

ROBERTS, H. A. 1981. Seed Banks in Soils. Advances in Applied Biology, v.6, p.1-55.

SABOGAL, C. 1992. Regeneration of tropical dry forest in Central America, with examples from Nicaragua. Jounal of Vegetation Science, v.3, p.407416.

SANTOS, S. L. 2000. Influência da serrapilheira na germinação e desenvolvimento de plântulas da Mata de Santa Genebra. 71p. Tese (Mestrado em Biologia Vegetal). Universidade Estadual de Campinas. Campinas, SP.

SANTOS, J. C.; LEAL, I. R.; ALMEIDA-CORTEZ, J. S.; FERNANDES, G. W.; TABARELLI, M. 2011. Caatinga: the scientific negligence experienced by a dry tropical forest. Tropical Conservation Science, v.4, n.3, p.276-286. 
Journal of Environmental Analysis and Progress V. 01 N. 01 (2016) 61-69

STATSOFT, Inc, 2003. Statistica for Windows (Data Analysis Software System), Version7.0. StatSoft, Inc., USA.

SCHULZ, K.; VOIGT, K.; BEUSCH, C.; ALMEIDA-CORTEZ, J. S.; KOWARIK, I.; WALZ, A.; CIERJACKS, A. 2016. Grazing deteriorates the soil carbon stocks of Caatinga forest ecosystems in Brazil. Forest Ecology and Management, v.367, p.62-70.
SIMPSON, R. L; LECK, M. A.; PARKER, V. T. 1989. Ecology of Soil Seed Banks. California: Academic Press. 385 p.

SOUZA, P. A.; VENTURIN, N.; GRIFFITH, J. J.; MARTINS, S. V. 2006. Avaliação do banco de sementes contido na serrapilheira de um fragmento florestal visando recuperação de áreas degradadas. Revista Cerne, Lavras, v.2, n.1, p.56-67. 